planner. What a paper delight are those flow charts of responsibility and accountability. Going from paper to practice is another matter. The ingredients that go to making a group work well together are easier to recognise than to describe. Committees fail if a member arrives saying to himself ' $\mathrm{I}$ am a doctor, she is a nurse, he is an administrator'. The small group can work better because it is more likely that each member will arrive as himself or herself. Things break down when we assume representation of a group; then beware the masked man. Within the confines of a hospital it is worth making every effort to eliminate the number of times that people meet to debate problems of which they have only hearsay knowledge.

\title{
The Future of Academic Medicine
}

Departments of academic medicine in the UK are feeling as if a dark cloud is covering them. The term 'academic' was once defined as 'sceptical, scholarly'; now the O.E.D. defines it as 'unpractical and not leading to a decision'! When times are hard, 'unpractical' is regarded as 'unnecessary', and the recent financial cuts imposed by Government must mean that there is a widespread belief that academics may not be very necessary.

University clinical departments were established in the UK in 1912, to improve the scientific quality of the medicine practised throughout the hospital and community. Individuals in such a department were supposed to practise medicine, teach students, and advance the subject by academic research. Although it has been difficult to remain competent in all three areas, 'academics' in the UK have tried, with varying degrees of success. Many young doctors have spent a period of their postgraduate education in such departments, being given the opportunity to start new cross-disciplinary areas of clinical research and practice while being instilled with the spirit of enquiry. Many consultants in the NHS have had this type of education and are now to be found throughout the UK.

The really difficult problem with a university clinical department is establishing the right balance between the three areas that have to be covered. Since patient care must come first, it can, if in sufficient amount, destroy both teaching and research. One of the best ways of achieving a balance has been to have as many clinicians as possible within such a department, so that a balance can be achieved for each individual. Not surprisingly, this has given rise to the accusation of 'empire building'.

Why then do such departments consider themselves under threat of destruction? The reasons, I think, in order of importance, are as follows-

1. The effect of University Grants Committee financial cuts and the consequent reduction in the staff/student ratio must mean that it will become impossible to maintain the delicate balance between patient care, teaching and research. Research will suffer, and teaching, unless it becomes more efficient, will deteriorate in quantity if not in quality.

2. The attitudes of young people to academic research and the spirit of enquiry are generally negative. The attitudes are probably part of a general disillusionment with scientific enquiry and typical of the age, combined with an obsession with the overriding importance of caring for patients and doing things to them. There seems to be a widespread lack of interest in spending time and effort on establishing the value of many of the things that are done to patients, and little understanding that medicine is still practised in an ocean of ignorance. There is very little evidence that young people want to train to ensure that they do not become victims of the best advertiser.

3. The decision to spend a time in academic medicine causes serious financial difficulties. The payment of UMTs is patchy, and there are no moving expenses. For individuals of consultant status there is no right to earn an extra 10 per cent in private practice.

4. There are serious educational difficulties. The publication of training programmes by SACs and the demand that training posts should offer a balanced experience have made it difficult for doctors to follow an unconventional programme in the first ten years after qualification. Potential applicants for academic posts do not know that most SACs try to help and often approve a research post. Young doctors fear to stray from the path of conventional registrar and senior registrar training.

5. Our university clinical departments have been accused of (a) investigating patients to provide the staff with occupational therapy, and permitting the scientific approach to inhibit compassion; (b) neglecting preventive medicine and not being concerned with the application of what is already known; and (c) neglecting clinical strategy, health services research, and health policy (the relationship between ethics, medicine and law).

In my opinion there is a grain of truth in (a) and (b); we must focus on only doing creative research with clearly defined protocols, and we ought to be concerned with the practical implementation of research results, be they positive or negative. I accept the accusation in (c) but it takes time to establish the necessary principles of creative research in those areas that cross many disciplines.

In recent years there have been gloomy reports of the medical academic situation in the European and American literature. Thus, although we have our special problems in the UK, the problem is worldwide in nature. The decision by the Royal College of Physicians to set up a working party to discuss the problems facing academic medicine is to be commended. The results of its deliberations may result in the preservation of this aspect of clinical life of which we have, with good reason, been proud.

A. GuZ 